\title{
Restoring public trust in scientific research by reducing conflicts of interest
}

\author{
Arthur M. Feldman' and Douglas L. Mann ${ }^{2}$ \\ ${ }^{1}$ Lewis Katz School of Medicine at Temple University, Philadelphia, Pennsylvania, USA. ²Washington University School of Medicine, St. Louis, Missouri, USA.
}

c linician investigators and biomedical scientists share a fundamental obligation to produce new knowledge that can lead to better treatments or cures for human disease. Conflicts of interest (COIs) occur when professional responsibilities and altruistic goals are influenced by an outside interest that biases clinical judgement or interpretation of data. The public exposure of a failure to disclose COIs in clinical or translational research can lead to a loss of confidence in the medical profession and in scientific discovery, respectively. The Sunshine Act was seen as mitigating the lack of transparency in $\mathrm{COI}$ reporting by requiring the pharmaceutical and device industries to disclose all payments to physicians and teaching hospitals through the Open Payment program, a public and searchable database of industry payments. This database has certainly helped, but problems persist, as illustrated by front-page articles in the New York Times describing how undeclared COIs have roiled two major areas of medical research.

The first New York Times reports detailed the failure of a top cancer researcher and senior administrator at Memorial Sloan Kettering Cancer Center to disclose over \$3.0 million in compensation from industry (1). A follow-up report noted that this high level of remuneration was not unusual for influential physicians who serve on industry advisory boards (2). The second series of articles focused on a former Harvard physician-scientist and stem cell biologist who had failed to disclose his association with a biotechnology company (3). After a five-year review, Harvard Medical School and Brigham and Women's Hospital (HMS-BWH) called for the retraction of 31 of his studies due, not to COIs, but to serious concerns regarding the integrity of data showing, in part, that c-kit ${ }^{+}$stem cells could regenerate damaged heart (4). These two very different but equally high-profile events provide useful case studies of the adverse consequences that COIs can have on the integrity of research and can help guide development of improved reporting and oversight structures.

First, the Memorial Sloan Kettering case points out how academic institutions and professional associations often turn a blind eye to tracking and quantifying COIs, particularly when the conflict is held by a senior leader or a well-funded investigator. A failure to look for COIs and to appropriately regulate them is all too common across many professional societies. For example, the published policies of the National Comprehensive Cancer Network (NCCN) stipulate that authors of NCCN clinical guidelines may not have financial conflicts with the pharmaceutical industry that exceed a single payment of $\$ 20,000 /$ year or aggregate payments of greater than $\$ 50,000 /$ year (5). A recent study found, however, that $86 \%$ of guideline authors had at least one conflict and that eight of 125 authors had received payments over the benchmark levels (6). Societies have also failed to establish useful regulations for members of guideline committees. For example, the NCCN precludes members of guideline-writing committees from voting when they have a COI, but allows the conflicted members to stay in the room during those discussions at the discretion of the chair (5). In some cases, the conflicted panel members are even allowed to participate in the discussion. The NCCN

Conflict of interest: AMF is the scientific founder and has significant equity in and serves as a director of Renovacor Inc., which has in-licensed pending patents 15/115,807, 15/753,003, and 62/764,894 from Temple University. DLM is a member of the scientific advisory board of Tenya Therapeutics, for which he received an amount of compensation consistent with a potential conflict.

Copyright: @ 2019, American Society for Clinical Investigation.

Reference information: J Clin Invest. 2019;129(10):3971-3973. https://doi.org/10.1172/JCI131448.

guideline committees also need only have $51 \%$ of its members to be free of conflicts, raising the concern that a panel in which nearly half its members are conflicted would be hard-pressed to avoid bias.

The Harvard/Brigham and Women's Hospital case demonstrates a second limitation of the Open Payment system - that it does not capture payments made to physician-scientists who do not maintain a medical license or to nonphysician investigators. Furthermore, companies that do not yet produce an FDA-approved product need not report payments to researchers. The Open Payment system also does not track the number of relevant patents that an investigator has filed with the US patent office, an important metric because it marks the intent of a faculty member to commercialize intellectual property at a later point in time. Thus, while current COI policies capture the "conflict" of a practitioner who attends a dinner sponsored by a pharmaceutical company, they fail to identify substantive conflicts among investigators who are leading clinical trials that might alter the care of patients.

A third flaw in COI policies at many academic institutions is a failure to properly oversee participation in clinical trials by investigators who have commercialized their intellectual property and, as a result, have equity in the study sponsor. An absence of oversight often leads to investigators participating in clinical studies that lack rigor in trial design, data collection, and/or are prone to overinterpretation of "encouraging" data buried in clinical endpoints that were not prespecified. In this regard, two recent reviews on the use of cardiac stem cells for regenerating heart muscle pointed out that recent stem cell trials were rife with COIs $(7,8)$. For example, studies published in high-impact journals by investigators having an equity interest in the sponsoring entity or in a collaborating or contributing company reported salutary benefits of cell therapy in clinical trials that 


\section{Recommendations for improving disclosure and oversight of conflicts}

1. Published disclosures of COls could be more meaningful if they divulged the amount of money received from relevant commercial entities. When possible, authors should reconcile these numbers with the Open Payment database.

2. Ownership in a private or public biotechnology company should be included in all disclosures as well as the value of the equity or the percentage of issued stock that is held.

3. Published patent applications filed with the US patent office should be included as potential COIs.

4. Guideline- and consensus-writing committees should exclude members with COls or ask them to resolve their COls before joining the committee. When that is not possible, those with cOls should be excused from the room during relevant discussions. The time-honored excuse that the best clinicians consult for every company because their opinions are so highly valued and that they therefore should not be excluded from writing committees is not a valid reason for ignoring the problems inherent with COls.

5. A physician holding equity in a private or public biotechnology company or who serves as a director of a company should not participate as an investigator in any clinical trial sponsored by that company nor be a lead author on academic publications that report the study results. This is already a mandate at some, but far too few, academic centers in the US, and even when present it may be ignored.

6. Wherever possible, investigational new drug-enabling preclinical studies should be carried out by a clinical research organization and data analysis performed by an independent statistician, not by a trainee in the principal investigator's laboratory.

7. Journal editors as well as guest editors must be free of all COIs, including equity and patents. Publication committees should raise questions when there is an imbalance in the types or outcomes of studies published in their own journal as compared with those published by competitors. Furthermore, lead editors should be judicious in submitting research articles to their own journal, limiting their submissions as far as possible to editorials and policy statements.

8. Academic institutions, publishers, and professional organizations must all share in the responsibility to ensure that $\mathrm{COIs}$ are appropriately reported, fairly adjudicated, and dealt with in a consistent manner.

enrolled fewer than 20 patients per treatment group (9-11), lacked concordance in critical baseline demographic data (10), did not include a control group $(9,12)$, and/ or reported efficacy in the absence of a measured change in ventricular function (11). In fact, a randomized clinical trial that demonstrated a benefit of cardiac stem cells was subsequently retracted by the publisher because of questions regarding the validity of the data (13).

Finally, professional societies that support journals bear responsibility for making sure that the editors and reviewers are made aware of all author COIs before publishing consensus documents. This is illustrated by a 2017 consensus statement on cardiomyocyte regeneration. The authors concluded that bone marrow-derived "c-kit ${ }^{+}$cells or mesenchymal stem cells may confer structural or functional benefits" (14). Half of the authors disclosed an equity interest in a related biotechnology company, one author failed to note that he had founded a stem cell company, and a second author did not disclose that he had filed multiple stem cell patents, information that might have given pause to the reviewers and the editors if it were known during the review process.

How can academic institutions, professional associations, and publishers redefine the ground rules for the disclosure and oversight of COIs in order to stem this growing problem? We propose that a few simple rules could go a long way toward increasing the public's trust, as detailed in Recommendations for improving disclosure and oversight of conflicts.

The growing number of reported COIs and our failure to properly manage them threatens the integrity of the scientific process insofar as it has the potential to bias scientific communications, which in turn will erode public trust in the scientific community. This statement notwithstanding, COI guidelines must not be so restrictive that they shackle academic freedom and stifle the entrepreneurial spirit of investigators. The scientific community must seek a transparent middle ground. The guidelines proposed herein may be viewed as too strident by some and too lenient by others, but they will hopefully serve as a starting point for national discussions with the goal of deriving new and innovative policies that are fair but also enforceable.

Address correspondence to: Arthur M. Feldman, Department of Medicine, Division of Cardiology, Lewis Katz School of Medicine at Temple University, 3440 North Broad Street, Kresge Building, Suite 200, Philadelphia, Pennsylvania 19140, USA. Phone: 215.707.1673 ; Email: arthur. feldman@tuhs.temple.edu.

1. Ornstein C, Thomas K. Top cancer researcher fails to disclose corporate financial ties in major research journals. New York Times. September 9, 2018.

2. Thomas K, Ornstein C. When doctors serve on company boards. New York Times. December 31, 2018.

3. Leri A, Rota M, Pasqualini FS, Goichberg P, Anversa P. Origin of cardiomyocytes in the adult heart. Circ Res. 2015;116(1):150-166.

4. Kolata G. Harvard calls for retraction of dozens of studies by noted cardiac researcher. New York Times. November 15, 2018.

5. Network NCC. NCCN disclosure policies and potential conflicts of interest. http://www.nccn. org/about/disclosure.aspx.

6. Mitchell AP, Basch EM, Dusetzina SB. Financial relationships with industry among National Comprehensive Cancer Network guideline authors. JAMA Oncol. 2016;2(12):1628-1631.

7. Chien KR, Frisén J, Fritsche-Danielson R, Melton DA, Murry CE, Weissman IL. Regenerating the field of cardiovascular cell therapy. Nat Biotechnol. 2019;37(3):232-237.

8. Epstein JA. A time to press reset and regenerate cardiac stem cell biology [published online ahead of print November 27, 2018]. JAMA Cardiol. https://doi.org/10.1001/jamacardio.2018.4435.

9. Florea V, et al. Dose comparison study of allogeneic mesenchymal stem cells in patients with ischemic cardiomyopathy (The TRIDENT Study). Circ Res. 2017;121(11):1279-1290.

10. Bartolucci J, et al. Safety and efficacy of the intravenous infusion of umbilical cord mesenchymal stem cells in patients with heart 
failure: a phase $1 / 2$ randomized controlled trial (RIMECARD Trial [Randomized Clinical Trial of Intravenous Infusion Umbilical Cord Mesenchymal Stem Cells on Cardiopathy]). Circ Res. 2017;121(10):1192-1204.

11. Butler J, et al. Intravenous allogeneic mesenchymal stem cells for nonischemic car- diomyopathy: safety and efficacy results of a phase ii-a randomized trial. Circ Res. 2017;120(2):332-340.

12. Tompkins BA, et al. Comparison of mesenchymal stem cell efficacy in ischemic versus nonischemic dilated cardiomyopathy. J Am Heart Assoc. 2018;7(14):e008460.
13. Bolli R, et al. Cardiac stem cells in patients with ischaemic cardiomyopathy (SCIPIO): initial results of a randomised phase 1 trial. Lancet. 2011;378(9806):1847-1857.

14. Eschenhagen T, et al. Cardiomyocyte regeneration: a consensus statement. Circulation. 2017;136(7):680-686. 